\title{
Comparative Assessment of Predictive Performance of PRECISE-DAPT, CRUSADE, and ACUITY Scores in Risk Stratifying 30-Day Bleeding Events
}

Hideyuki Kawashima ${ }^{1,2}$ Chao Gao ${ }^{2,3}$ Kuniaki Takahashi ${ }^{1}$ Mariusz Tomaniak ${ }^{4}$ Masafumi Ono ${ }^{1,2}$ Hironori Hara ${ }^{1}$ Rutao Wang ${ }^{2,3}$ Ply Chichareon $^{5}$ Harry Suryapranata $^{3}$ Simon Walsh $^{6}$ James Cotton ${ }^{7}$ Rene Koning ${ }^{8}$ Benno Rensing ${ }^{9}$ Joanna Wykrzykowska ${ }^{1}$ Robbert J. de Winter ${ }^{1}$ Scot Garg ${ }^{10}$ Richard Anderson ${ }^{11}$ Christian Hamm ${ }^{12}$ Philippe Gabriel Steg ${ }^{13,14}$ Yoshinobu Onuma ${ }^{2}$ Patrick W. Serruys 2,15

1 Department of Clinical and Experimental Cardiology, Amsterdam Cardiovascular Sciences, Amsterdam UMC, University of Amsterdam, Heart Center, Amsterdam, The Netherlands

2 Department of Cardiology, National University of Ireland, Galway (NUIG), Galway, Ireland

3 Department of Cardiology, Radboudumc, Nijmegen, The Netherlands

${ }^{4}$ First Department of Cardiology, Medical University of Warsaw,

Warsaw, Poland

${ }^{5}$ Cardiology Unit, Department of Internal Medicine, Faculty of Medicine, Prince of Songkla University, Songkhla, Thailand

${ }^{6}$ Department of Cardiology, Belfast Health and Social Care Trust, Belfast, United Kingdom

${ }^{7}$ Queen Elizabeth Hospital Birmingham, Birmingham, United Kingdom

${ }^{8}$ Clinique Saint-Hilaire, Rouen, France

${ }^{9}$ Sint-Antonius Ziekenhuis, Nieuwegein, The Netherlands
Address for correspondence Patrick W. Serruys, MD, PhD, FESC, FACC, National University of Ireland, Galway (NUIG), University Road, Galway H91 TK33, Ireland (e-mail: patrick.w.j.c.serruys@gmail.com).

10 East Lancashire Hospitals NHS Trust, Blackburn, Lancashire, United Kingdom

${ }^{11}$ Cardiff and Vale University Health Board, Wales, United Kingdom

${ }^{12}$ Kerckhoff Heart Center, Campus University of Giessen, Bad Nauheim, Germany

${ }^{13}$ Assistance Publique-Hôpitaux de Paris, INSERM U-1148, FACT (French Alliance for Cardiovascular Trials), Hôpital Bichat, Université de Paris, Paris, France

14 Royal Brompton Hospital, National Heart and Lung Institute, Imperial College London, London, United Kingdom

${ }^{15} \mathrm{NHLI}$, Imperial College London, London, United Kingdom

Thromb Haemost 2020;120:1087-1095.

Abstract
Keywords
- bleeding scores
- major bleeding
- percutaneous
coronary intervention
- dual-antiplatelet
therapy
- discrimination
- calibration

Background The utility of the PRECISE-DAPT score in predicting short-term major bleeding, either alone, or in comparison with the CRUSADE and ACUITY scores, has not been investigated. This analysis compared the predictive performances of the three bleeding scores in stratifying the risk of 30-day major bleeding postpercutaneous coronary intervention in patients with dual-antiplatelet therapy.

Methods In this post hoc subanalysis of the GLOBAL LEADERS trial, the primary safety objective (bleeding according to the Bleeding Academic Research Consortium [BARC] criteria [type 3 or 5]) was assessed at 30 days according to the three scores in the overall population, and in patients with acute (ACS) and chronic coronary syndrome (CCS). Results In a total of 15,968 patients, we calculated all three scores in 14,709 (92.1\%). Irrespective of clinical presentation, the PRECISE-DAPT (c-statistics: 0.648, 0.653, and 0.641, respectively), CRUSADE (c-statistics: $0.641,0.639$, and 0.644 , respectively), and ACUITY (cstatistics: $0.633,0.638$, and 0.623 , respectively) scores were no significant between-score received

February 22, 2020

accepted after revision

April 19, 2020
DOI https://doi.org/

$10.1055 / \mathrm{s}-0040-1712449$. ISSN 0340-6245. (c) 2020. The Author(s).

This is an open access article published by Thieme under the terms of the Creative Commons Attribution-NonDerivative-NonCommercial-License, permitting copying and reproduction so long as the original work is given appropriate credit. Contents may not be used for commercial purposes, or adapted, remixed, transformed or built upon. (https://creativecommons.org/ licenses/by-nc-nd/4.0/)

Georg Thieme Verlag KG, Rüdigerstraße 14, 70469 Stuttgart, Germany 
differences in discriminatory performance for BARC 3 or 5 bleeding up to 30 days, and similarly the PRECISE-DAPT score had a comparable discriminative capacity according to the integrated discrimination improvement when compared with the other scores. In ACS, the CRUSADE score had a poor calibration ability (Hosmer-Lemeshow goodness-of-fit [GOF] chi-square $=15.561, p=0.049$ ), whereas in CCS, the PRECISE-DAPT score had poor calibration (GOF chi-square $=15.758, p=0.046$ ).

Conclusion The PRECISE-DAPT score might be clinically useful in the overall population and ACS patients for the prediction of short-term major bleeding considering its discriminative and calibration abilities.

\section{Introduction}

Bleeding is a common adverse event after percutaneous coronary intervention (PCI) and is associated with increased morbidity and mortality. ${ }^{1,2}$ Bleeding predictors have been described extensively; they are related mostly to the patient's clinical characteristics, the invasiveness of the procedure, and the potency of the antithrombotic regimen. In particular, the potency and duration of dual-antiplatelet therapy (DAPT) after PCI are mainly based on the patient's clinical presentation (acute [ACS] or chronic coronary syndromes [CCS]) and the patient's bleeding risk. ${ }^{3,4}$ To date, some bleeding risk scores have been validated for the prediction of early and late bleeding events.

The CRUSADE (Can Rapid Risk Stratification of Unstable Angina Patients Suppress Adverse Outcomes With Early Implementation of the American College of Cardiology/American Heart Association Guidelines) and ACUITY (Acute Catheterization and Urgent Intervention Triage Strategy) bleeding risk scores $^{5,6}$ have been developed to estimate the baseline risk for short-term major bleeding. ${ }^{7-10}$ Historically, the CRUSADE score was designed for non-ST elevation myocardial infarction (STEMI) population, whereas the ACUITY score was derived from ACS population. Recently, the PRECISE-DAPT (Predicting Bleeding Complication in Patients Undergoing Stent Implantation and Subsequent Dual Antiplatelet Therapy) score has been developed from the PLATO trial (ACS population) and Bern PCI registry (all-comers population). ${ }^{11}$ Despite the development of various bleeding scores, estimation of individual bleeding risk remains a clinical challenge and each bleeding risk score might be more accurate in the clinical scenario from which the scores were designed. Choi et al ${ }^{12}$ have validated the PRECISE-DAPT, CRUSADE, and ACUITY scores in an all-comers population and showed that all three scores had a good predictive performance for 1-year bleeding events. The primary endpoint in the PRECISE-DAPT trial ${ }^{11}$ was bleeding up to 12 months after the index PCI procedure, and therefore the utility of the PRECISEDAPT score in predicting short-term bleeding events post-PCI in patients with DAPT, either alone, or in comparison with the CRUSADE and ACUITY scores, has not yet been investigated.

Our study sought to evaluate and compare the performances of the PRECISE-DAPT, CRUSADE, and ACUITY scores for predicting 30-day major bleeding post-PCI in patients with DAPT in the overall population of the GLOBAL LEADERS trial, as well as in patients with ACS and CCS. ${ }^{13}$

\section{Methods}

\section{Study Population}

This article is a post hoc analysis of the GLOBAL LEADERS trial, a multicenter, prospective, and open-label randomized controlled trial (NCT01813435). ${ }^{14,15}$ Details of the study design and protocol have been reported elsewhere. ${ }^{16}$ In brief, the present study enrolled 15,991 patients at 130 hospitals in 18 countries between July 2013 to November 2015 in an "allcomers" design: no restriction regarding the clinical presentation of patients, the complexity of lesions, or the number of stents used. Twenty-three patients withdrew consent and requested data deletion from the database, leaving 15,968 patients in the present analysis. The trial randomly assigned patients before PCI to either (1) the experimental strategy with 1-month DAPT (aspirin and ticagrelor) followed by 23month ticagrelor monotherapy, or (2) the reference regimen with 12-month DAPT (aspirin and either ticagrelor for ACS or clopidogrel for CCS followed by 12-month aspirin monotherapy, respectively. Of note, patients with planned oral anticoagulation were excluded. All types of anatomic lesions were included and treated by default with Biolimus A9eluting stents (BioMatrix, Biosensors, Europe) of which the use was unrestricted in number, length, and diameter.

The trial was approved by the institutional review board at each center and followed the ethical principles of the Declaration of Helsinki. All the patients gave written informed consent prior to participation in the trial. Patients who had any missing variables for the calculation of any score were excluded from this analysis, and as the number was small, there was no requirement for imputation. ${ }^{17}$

\section{Variable Definition}

The PRECISE-DAPT, ${ }^{11}$ CRUSADE, $^{5}$ and ACUITY $^{6}$ scores were derived from the patients' clinical characteristics recorded at the time of enrolment into the study. The PRECISE-DAPT score was derived from five variables (age, creatinine clearance, hemoglobin, white blood cell count, and previous spontaneous bleeding). The CRUSADE score was derived from eight variables (female sex, diabetes mellitus, chronic heart failure, valvular heart disease, heart rate, systolic blood pressure, glomerular filtration rate, and hematocrit). The ACUITY score consists of seven variables (female sex, age, type of ACS: unstable angina, non-STEMI, or STEMI, serum creatinine, and white blood cell count; all analyzed as ordinal 
categories). Hemoglobin equals to 0,999.9, and less than 3.1 and white blood cell count $>30$ or equals to 0 were excluded and treated as missing value. The total scores for each patient were assessed using an online calculator (Sikuli app [http:// sikulix.com]) with all the prognostic variables included in the score.

\section{Study Objectives}

The primary objective was to compare the predictive performance of the PRECISE-DAPT, CRUSADE, and ACUITY scores for Bleeding Academic Research Consortium (BARC) 3 or 5 bleeding at 30 days post-PCI in the overall population and in patients with ACS and CCS. In the GLOBAL LEADERS trial, bleeding events based on the BARC criteria were sitereported, and were the only bleeding criteria used in this trial. No other secondary endpoints were assessed in this study.

\section{Statistical Analysis}

Quantitative variables are reported as mean \pm standard deviation or median and interquartile range. Qualitative variables are expressed as numeric values and percentages. The discriminative capacities of the three scores were assessed with $c$ statistics and they were compared using the DeLong test. ${ }^{18} \mathrm{~A}$ $p$-value of $<0.05$ was considered statistically significant. Recent expert opinion refers to a $c$-statistics $<0.60$ as poor discrimination; 0.60 to 0.75 as possibly helpful discrimination; and more than 0.75 as clearly useful discrimination. ${ }^{19}$ The discriminative capacities of the three scores were also compared by integrated discrimination improvement (IDI). ${ }^{20}$ In addition, relative IDI, which defined as the increase in discrimination slopes divided by the slope of the old model, were calculated to clarify the justification of IDI. ${ }^{21}$ The discrimination slope, which was defined as the slope of a linear regression of predicted probabilities of an event derived from a prognostic model on the binary event status, has recently gained popularity as a measure of model performance. ${ }^{22}$ The calibration of the models was evaluated using the Hosmer-Lemeshow goodness-of-fit (GOF) statistical test. ${ }^{23}$ A significant $p$-value less than 0.05 indicated a poor calibration. All data were processed using SPSS version 26.0 (IBM Inc, Armonk, New York, United States) and R version 3.6.0 (R Foundation for Statistical Computing, Vienna, Austria).

\section{Results}

\section{Baseline Characteristics}

Baseline characteristics in the present study are shown in -Table 1. In the GLOBAL LEADERS trial, complete data to calculate the PRECISE-DAPT, CRUSADE, and ACUITY scores were available in 14,928patients (93.5\%), 15,054 patients (94.3\%), and 14,853 patients (93.0\%), respectively. The 1,259 patients (7.9\%) for whom the scores could not be calculated due to missing values were excluded from this analysis. Therefore, we calculated all three scores in 14,709 patients (92.1\%) and those patients were analyzed in the present study. The mean value \pm standard deviation of the PRECISEDAPT score in the overall population was $16.4 \pm 8.8$,
Table 1 Patient characteristics

\begin{tabular}{|c|c|}
\hline Age, $\mathrm{y} \pm$ standard deviation & $64.6 \pm 10.3$ \\
\hline Body mass index, $\mathrm{kg} / \mathrm{m}^{2}$ & $28.2 \pm 4.6$ \\
\hline Male & $11,289 / 14,709(76.7 \%)$ \\
\hline Female & $3,420 / 14,709$ (23.3\%) \\
\hline \multicolumn{2}{|l|}{ Medical history } \\
\hline Diabetes mellitus & $3,748 / 14,709$ (25.5\%) \\
\hline $\begin{array}{l}\text { Insulin-dependent } \\
\text { diabetes mellitus }\end{array}$ & $1,123 / 14,709$ (7.6\%) \\
\hline Hypertension & $10,904 / 14,709$ (74.1\%) \\
\hline Hyperlipidemia & $9,977 / 14,709$ (67.8\%) \\
\hline Previous stroke & $394 / 14,709$ (2.7\%) \\
\hline $\begin{array}{l}\text { Previous myocardial } \\
\text { infarction }\end{array}$ & $3,448 / 14,709$ (23.4\%) \\
\hline $\begin{array}{l}\text { Previous percutaneous } \\
\text { coronary intervention }\end{array}$ & $4,850 / 14,709(33.0 \%)$ \\
\hline $\begin{array}{l}\text { Previous coronary artery } \\
\text { bypass grafting }\end{array}$ & $866 / 14,709$ (5.9\%) \\
\hline Peripheral vascular disease & $936 / 14,709(6.4 \%)$ \\
\hline $\begin{array}{l}\text { Chronic obstructive } \\
\text { pulmonary disease }\end{array}$ & $761 / 14,709$ (5.2\%) \\
\hline Previous major bleeding & $89 / 14,709(0.6 \%)$ \\
\hline Current smoker & $3,864 / 14,709$ (26.3\%) \\
\hline Impaired renal failure & $2,026 / 14,709(13.8 \%)$ \\
\hline \multicolumn{2}{|l|}{ Clinical presentation } \\
\hline Chronic coronary syndrome & $7,653 / 14,709$ (52.0\%) \\
\hline Acute coronary syndrome & $7,056 / 14,709(48.0 \%)$ \\
\hline Unstable angina & $1,927 / 14,709$ (13.1\%) \\
\hline $\begin{array}{l}\text { Non-ST-elevation } \\
\text { myocardial infarction }\end{array}$ & $3,189 / 14,709$ (21.7\%) \\
\hline $\begin{array}{l}\text { ST-elevation } \\
\text { myocardial infarction }\end{array}$ & $1,940 / 14,709$ (13.2\%) \\
\hline \multicolumn{2}{|l|}{ Access site } \\
\hline Radial & $10,765 / 14,709(73.2 \%)$ \\
\hline Brachial & $19 / 14,709(0.1 \%)$ \\
\hline Femoral & $3,925 / 14,709(26.7 \%)$ \\
\hline \multicolumn{2}{|c|}{ Dual antiplatelet therapy (aspirin with) } \\
\hline Ticagrelor & $7,347 / 14,709$ (49.9\%) \\
\hline Clopidogrel & $7,362 / 14,709$ (50.1\%) \\
\hline \multicolumn{2}{|l|}{ Bleeding risk scores } \\
\hline PRECISE-DAPT score & $16.4 \pm 8.8$ \\
\hline CRUSADE score & $20.5 \pm 12.2$ \\
\hline ACUITY score & $8.8 \pm 7.1$ \\
\hline
\end{tabular}

Abbreviations: ACUITY, Acute Catheterization and Urgent Intervention Triage Strategy; CRUSADE, Can Rapid Risk Stratification of Unstable Angina Patients Suppress Adverse Outcomes With Early Implementation of the American College of Cardiology/American Heart Association Guidelines; PRECISE-DAPT, Predicting Bleeding Complication in Patients Undergoing Stent Implantation and Subsequent Dual Antiplatelet Therapy. Note: Values are expressed as $n(\%)$ or mean \pm standard deviation. 


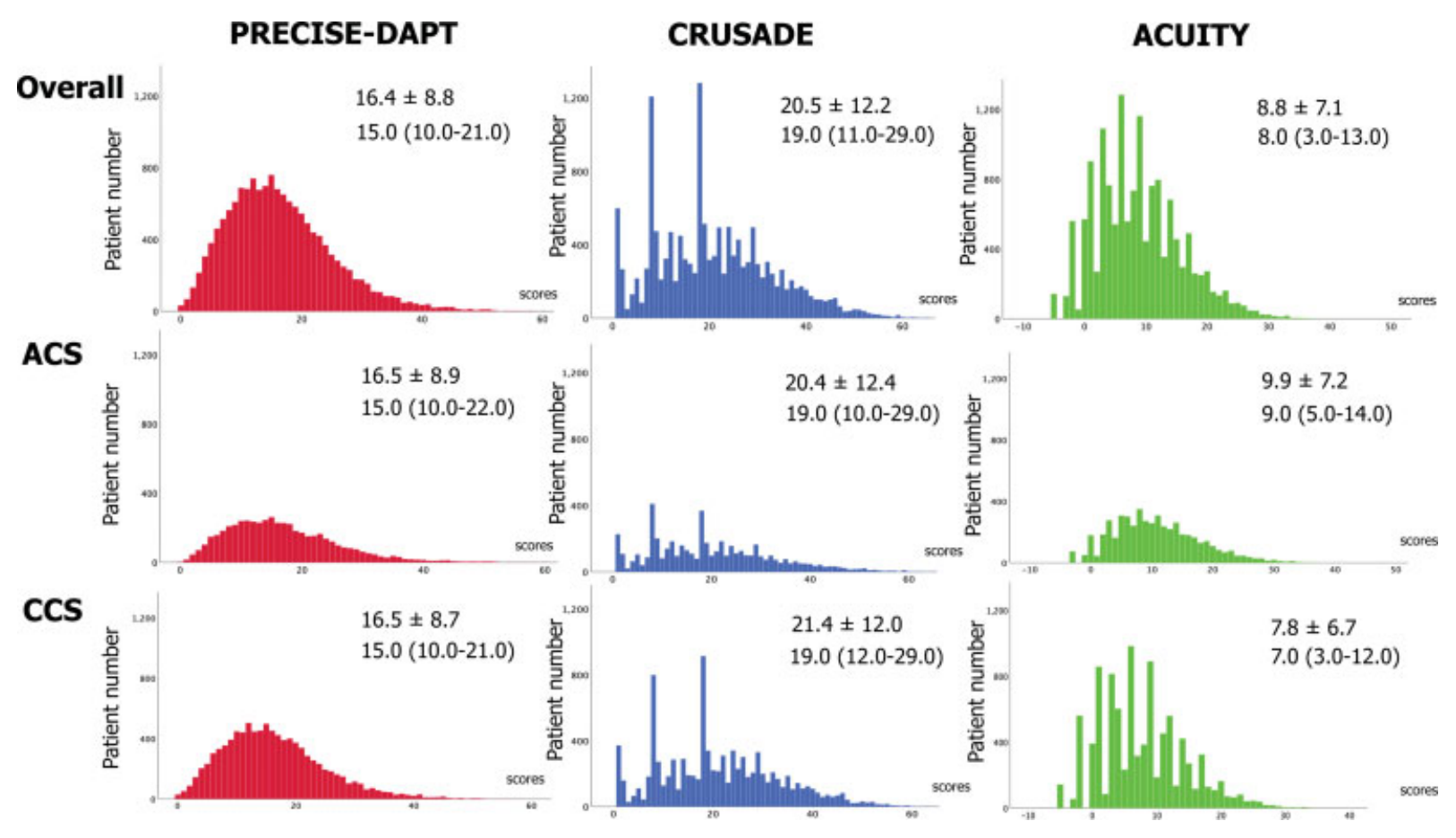

Fig. 1 Histograms of PRECISE-DAPT, CRUSADE, and ACUITY score according to each clinical presentation. The red histograms show the PRECISE DAPT score, the blue are the CRUSADE score, and the green are the ACUITY score. The overall population is shown in the top of the figure, ACS patients are in the middle, and CCS patients are in the bottom. The three scores according to each clinical presentation are expressed as mean \pm standard deviation and median and interquartile range (IQR). ACS, acute coronary syndrome; ACUITY, Acute Catheterization and Urgent Intervention Triage Strategy; CCS, chronic coronary syndrome; CRUSADE, Can Rapid Risk Stratification of Unstable Angina Patients Suppress Adverse Outcomes With Early Implementation of the American College of Cardiology/American Heart Association Guidelines; PRECISE-DAPT, Predicting Bleeding Complication in Patients Undergoing Stent Implantation and Subsequent Dual Antiplatelet Therapy.

CRUSADE score was $20.5 \pm 12.2$, and the ACUITY score was $8.8 \pm 7.1$, respectively. The distribution of these scores according to each clinical presentation (the overall population, ACS patients, and CCS patients) is shown in - Fig. 1.

\section{Discrimination Capacities of the Three Risk Scores according to Each Clinical Presentation}

- Table 2 shows the comparison of discriminative capacities between the three risk scores by the DeLong test according to each clinical presentation. In the overall population, ACS patients, and CCS patients, respectively, the PRECISE-DAPT ( $c$ statistics: $0.648,0.653$, and 0.641 , respectively), CRUSADE ( $c$ statistics: $0.641,0.639$, and 0.644 , respectively), and ACUITY ( $c$ statistics: $0.633,0.638$, and 0.623 , respectively) scores all had possibly helpful discrimination abilities for BARC 3 or 5 bleeding, with no statistically significant differences between the scores.

The IDI and relative IDI between the three risk scores according to each clinical presentation are shown in -Table 3. In the overall population, the PRECISE-DAPT score had a comparable discriminative capacity for BARC 3 or 5 bleeding when compared with the other scores (PRECISEDAPT score vs. CRUSADE score [reference]: IDI $=0.10 \%$, $p=0.249$, PRECISE-DAPT score vs. ACUITY score [reference]: IDI $=0.11 \%, p=0.249$, and CRUSADE score vs. ACUITY score [reference]: IDI $<0.01 \%, p=0.959$, respectively).

In ACS patients, there was no significant difference in discrimination for BARC 3 or 5 bleeding.

In CCS patients, the PRECISE-DAPT score had a better discrimination for BARC 3 or 5 bleeding than the CRUSADE and ACUITY scores (reference) (IDI $=0.39 \%, p=0.017$ and IDI $=0.39 \%, p=0.032$, respectively).

\section{Calibration Abilities of the Three Risk Scores according to Each Clinical Presentation}

- Table 4 shows the calibration abilities of the three risk scores by the Hosmer-Lemeshow GOF test according to each clinical presentation. In the overall population, the three scores had acceptable calibration abilities for BARC 3 or 5 bleeding.

In ACS patients, the CRUSADE score had a poor calibration for BARC 3 or 5 bleeding (GOF chi-square $=15.561$ and $p=0.049$ ) (-Fig. 2).

In CCS patients, the PRECISE-DAPT score had a poor calibration for BARC 3 or 5 bleeding (GOF chisquare $=15.758, p=0.046)$.

\section{Discussion}

This is the first study to investigate the predictive performance of the PRECISE-DAPT score, in comparison with the CRUSADE and ACUITY scores, for 30-day major bleeding post-PCI in patients with DAPT using the GLOBAL LEADERS population. The main findings of this study can be summarized as:

1. Irrespective of clinical presentation, the PRECISE-DAPT, CRUSADE, and ACUITY scores had possibly helpful discriminative abilities (c-statistics: 0.60 to 0.75 ) for 30-day BARC 3 or 5 bleeding, with no statistically significant differences between the scores. 
Table 2 Comparison of discriminative capacities between the three risk scores by DeLong test according to each clinical presentation

\begin{tabular}{|c|c|c|c|c|c|c|}
\hline & PRECISE-DAPT score & CRUSADE score & ACUITY score & $\begin{array}{l}\text { PRECISE-DAPT } \\
\text { vs CRUSADE }\end{array}$ & $\begin{array}{l}\text { PRECISE-DAPT } \\
\text { vs ACUITY }\end{array}$ & $\begin{array}{l}\text { CRUSADE } \\
\text { vs ACUITY }\end{array}$ \\
\hline & c-statistics (95\% Cl) & c-statistics (95\% Cl) & c-statistics $(95 \% \mathrm{Cl})$ & $p$-Value & $p$-Value & $p$-Value \\
\hline \multicolumn{7}{|l|}{ Overall } \\
\hline BARC 3 or 5 & $0.648(0.617-0.679)$ & $0.641(0.609-0.672)$ & $0.633(0.603-0.664)$ & 0.531 & 0.223 & 0.549 \\
\hline BARC 5 & $0.701(0.617-0.786)$ & $0.694(0.609-0.779)$ & $0.696(0.612-0.780)$ & 0.854 & 0.875 & 0.941 \\
\hline BARC 3 & $0.639(0.607-0.671)$ & $0.637(0.605-0.670)$ & $0.629(0.598-0.660)$ & 0.861 & 0.397 & 0.514 \\
\hline \multicolumn{7}{|l|}{ ACS } \\
\hline BARC 3 or 5 & $0.653(0.611-0.695)$ & $0.639(0.596-0.683)$ & $0.638(0.597-0.678)$ & 0.406 & 0.343 & 0.915 \\
\hline BARC 5 & $0.683(0.576-0.790)$ & $0.708(0.592-0.823)$ & $0.701(0.595-0.807)$ & 0.652 & 0.646 & 0.862 \\
\hline BARC 3 & $0.646(0.602-0.690)$ & $0.638(0.592-0.683)$ & $0.632(0.590-0.674)$ & 0.605 & 0.385 & 0.744 \\
\hline \multicolumn{7}{|l|}{ CCS } \\
\hline BARC 3 or 5 & $0.641(0.596-0.687)$ & $0.644(0.599-0.689)$ & $0.623(0.577-0.668)$ & 0.876 & 0.265 & 0.184 \\
\hline BARC 5 & $0.726(0.588-0.865)$ & $0.676(0.551-0.800)$ & $0.671(0.528-0.815)$ & 0.251 & 0.212 & 0.895 \\
\hline BARC 3 & $0.631(0.583-0.678)$ & $0.639(0.592-0.686)$ & $0.619(0.572-0.665)$ & 0.633 & 0.498 & 0.240 \\
\hline
\end{tabular}

Abbreviations: ACS, acute coronary syndrome; ACUITY, Acute Catheterization and Urgent Intervention Triage Strategy; BARC, Bleeding Academic Research Consortium; CCS, chronic coronary syndrome; Cl, confidence interval; CRUSADE, Can Rapid Risk Stratification of Unstable Angina Patients Suppress Adverse Outcomes With Early Implementation of the American College of Cardiology/American Heart Association Guidelines; PRECISEDAPT, Predicting Bleeding Complication in Patients Undergoing Stent Implantation and Subsequent Dual Antiplatelet Therapy.

Table 3 Integrated discrimination improvement and relative integrated discrimination improvement for bleeding events between the three risk scores according to each clinical presentation

\begin{tabular}{|c|c|c|c|c|c|c|c|c|c|}
\hline & \multicolumn{3}{|c|}{ PRECISE-DAPT vs. CRUSADE } & \multicolumn{3}{|c|}{ PRECISE-DAPT vs. ACUITYa } & \multicolumn{3}{|c|}{ CRUSADE vs. ACUITYa } \\
\hline & IDI, \% & $p$-Value & rIDI, \% & IDI, \% & $p$-Value & rIDI, \% & IDI, \% & $p$-Value & rIDI, \% \\
\hline \multicolumn{10}{|l|}{ Overall } \\
\hline BARC 3 or 5 & 0.10 & 0.249 & 15.5 & 0.11 & 0.249 & 17.0 & $<0.01$ & 0.959 & $<0.01$ \\
\hline BARC 5 & -0.04 & 0.477 & -36.8 & -0.05 & 0.093 & -46.0 & -0.01 & 0.813 & -9.2 \\
\hline BARC 3 & 0.10 & 0.177 & 17.7 & 0.09 & 0.246 & 15.9 & -0.01 & 0.853 & $\begin{array}{ll}-1.8 \\
\end{array}$ \\
\hline \multicolumn{10}{|l|}{ ACS } \\
\hline BARC 3 or 5 & -0.08 & 0.390 & -9.3 & $<0.01$ & 0.967 & $<0.01$ & 0.09 & 0.263 & 10.4 \\
\hline BARC 5 & -0.16 & 0.040 & -125.4 & -0.09 & 0.027 & -70.6 & 0.07 & 0.124 & 54.9 \\
\hline BARC 3 & -0.04 & 0.573 & -5.1 & 0.02 & 0.770 & 2.6 & 0.06 & 0.313 & 7.7 \\
\hline \multicolumn{10}{|l|}{ CCS } \\
\hline BARC 3 or 5 & 0.39 & 0.017 & 87.8 & 0.39 & 0.032 & 87.8 & $<0.01$ & 0.998 & $<0.01$ \\
\hline BARC 5 & 0.08 & 0.200 & 87.5 & 0.02 & 0.611 & 21.9 & -0.07 & 0.385 & -76.5 \\
\hline BARC 3 & 0.35 & 0.019 & 46.6 & 0.34 & 0.056 & 60.8 & -0.02 & 0.861 & -5.5 \\
\hline
\end{tabular}

Abbreviations: ACS, acute coronary syndrome; ACUITY, Acute Catheterization and Urgent Intervention Triage Strategy; BARC, Bleeding Academic Research Consortium; CCS, chronic coronary syndrome; CRUSADE, Can Rapid Risk Stratification of Unstable Angina Patients Suppress Adverse Outcomes With Early Implementation of the American College of Cardiology/American Heart Association Guidelines; IDI, integrated discrimination improvement; PRECISE-DAPT, Predicting Bleeding Complication in Patients Undergoing Stent Implantation and Subsequent Dual Antiplatelet Therapy; rIDI, relative IDI.

${ }^{a}$ The model considered each bleeding risk score as a reference value for the others.

2. In the overall population and ACS patients, the PRECISEDAPT score had a similar discriminative capacity for BARC 3 or 5 bleeding according to the IDI when compared with the CRUSADE and ACUITY scores, and especially in CCS patients, the PRECISE-DAPT score had a better discrimination than the other scores. 
Table 4 Calibration abilities of the three risk scores by Hosmer-Lemeshow good-of-fit test according to each clinical presentation

\begin{tabular}{|c|c|c|c|c|c|c|}
\hline & \multicolumn{2}{|c|}{ PRECISE-DAPT } & \multicolumn{2}{|l|}{ CRUSADE } & \multicolumn{2}{|l|}{ ACUITY } \\
\hline & Chi-square & $p$-Value & Chi-square & $p$-Value & Chi-square & $p$-Value \\
\hline \multicolumn{7}{|l|}{ Overall } \\
\hline BARC 3 or 5 & 7.830 & 0.450 & 11.767 & 0.162 & 15.259 & 0.054 \\
\hline BARC 5 & 7.639 & 0.470 & 5.206 & 0.735 & 9.968 & 0.267 \\
\hline BARC 3 & 6.666 & 0.573 & 10.961 & 0.204 & 15.065 & 0.058 \\
\hline \multicolumn{7}{|l|}{ ACS } \\
\hline BARC 3 or 5 & 3.480 & 0.901 & 15.561 & 0.049 & 9.159 & 0.329 \\
\hline BARC 5 & 4.656 & 0.794 & 7.089 & 0.527 & 6.154 & 0.630 \\
\hline BARC 3 & 5.002 & 0.757 & 14.916 & 0.061 & 10.166 & 0.254 \\
\hline \multicolumn{7}{|l|}{ CCS } \\
\hline BARC 3 or 5 & 15.758 & 0.046 & 6.057 & 0.641 & 10.992 & 0.202 \\
\hline BARC 5 & 8.215 & 0.413 & 10.252 & 0.248 & 10.038 & 0.262 \\
\hline BARC 3 & 14.191 & 0.077 & 5.266 & 0.729 & 11.282 & 0.186 \\
\hline
\end{tabular}

Abbreviations: ACS, acute coronary syndrome; ACUITY, Acute Catheterization and Urgent Intervention Triage Strategy; BARC, Bleeding Academic Research Consortium; CCS, chronic coronary syndrome; CRUSADE, Can Rapid Risk Stratification of Unstable Angina Patients Suppress Adverse Outcomes With Early Implementation of the American College of Cardiology/American Heart Association Guidelines; PRECISE-DAPT, Predicting Bleeding Complication in Patients Undergoing Stent Implantation and Subsequent Dual Antiplatelet Therapy.

PRECISE-DAPT

$\mathrm{p}=\mathbf{0 . 9 0 1}$

$\%$

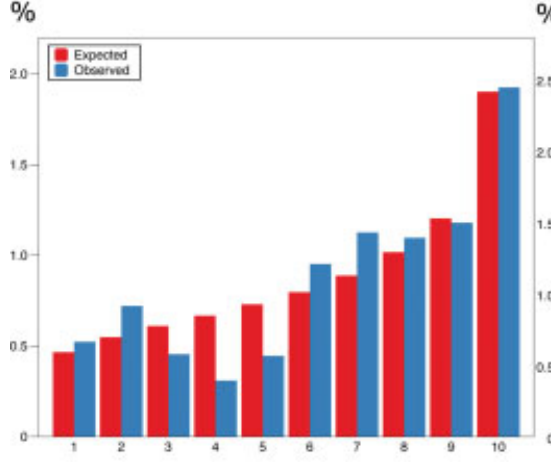

CRUSADE

$p=0.049$

$\%$

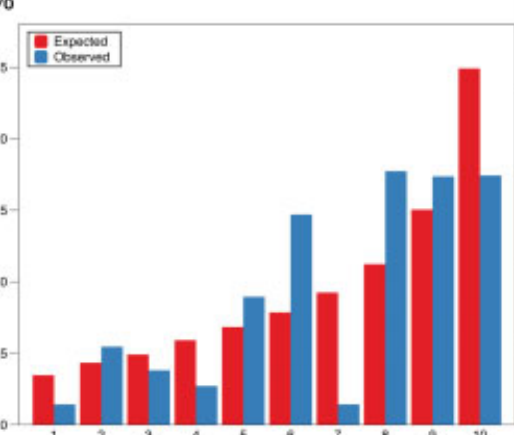

ACUITY

$p=0.329$

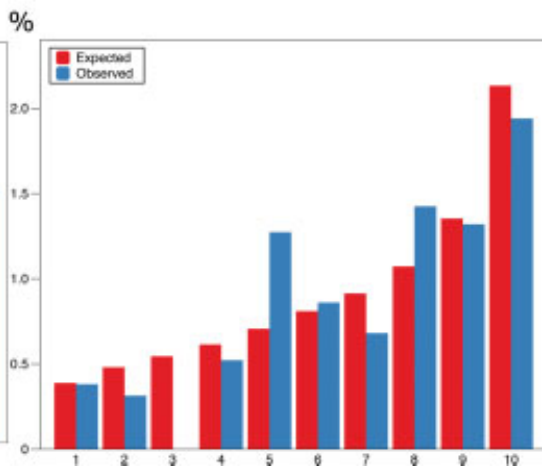

Fig. 2 Calibration capacity of PRECISE-DAPT, CRUSADE, and ACUITY score for BARC 3 or 5 bleeding up to 30 days in ACS patients. Calibration plots comparing the expected (red bar) and observed (blue bar) probabilities of BARC 3 or 5 bleeding. The left of the figure is the PRECISE-DAPT score, the center is the CRUSADE score, and the right is the ACUITY score. ACS, acute coronary syndrome; ACUITY, Acute Catheterization and Urgent Intervention Triage Strategy; BARC, bleeding according to the Bleeding Academic Research Consortium; CRUSADE, Can Rapid Risk Stratification of Unstable Angina Patients Suppress Adverse Outcomes With Early Implementation of the American College of Cardiology/American Heart Association Guidelines; PRECISE-DAPT, Predicting Bleeding Complication in Patients Undergoing Stent Implantation and Subsequent Dual Antiplatelet Therapy,.

3. The CRUSADE score had a poor calibration ability (GOF chi-square $=15.561$ and $p=0.049$ ) for BARC 3 or 5 bleeding in ACS patients, whereas the PRECISE-DAPT score had poor calibration (GOF chi-square $=15.758, p=0.046$ ) in CCS patients.

The CRUSADE and ACUITY scores were designed approximately 10 years ago, and currently no other newer bleeding risk stratification scores for predicting short-term bleeding in ACS patients exists. The patient population and medical treatment, including the choice of antiplatelet therapy, have changed considerably over the last decade. Notably, ticagrelor was not included in the armamentarium of antiplatelet therapy in these original trials; however, in the contemporary GLOBAL LEADERS trial, all ACS patients and patients with CCS in the experimental strategy received DAPT with ticagrelor for at least 1 month per protocol. Despite their historical derivation, recent data show that both the CRUSADE and ACUITY scores have equivalent capacity for the prediction of bleeding at 30 days after $\mathrm{PCI}$, even in patients with ACS receiving ticagrelor. ${ }^{9}$ However, the CRUSADE and ACUITY scores are rarely used in the routine practice and are 
suitable for patients without taking oral anticoagulation. ${ }^{5,6}$ The changes in interventional practice such as the use of radial access for coronary angiography and $\mathrm{PCI}$, and the shorter duration of DAPT might modify the predictive performance of bleeding risk scores. Importantly, the PRECISE-DAPT was developed in 2017 and was the most contemporary score and mostly driven by a prior history of bleeding. ${ }^{11}$

In the present study, the PRECISE-DAPT score showed a similar discriminative performance for major bleeding at 30 days compared with the CRUSADE and ACUITY scores in the overall population and ACS patients, and the CRUSADE score showed a poor calibration ability for 30-day major bleeding in ACS patients. Recent expert opinion described that discrimination and calibration are both important characteristics to evaluate the predictive performance of a risk model. ${ }^{19}$ Of note, the PRECISE-DAPT score includes previous bleeding as one of the components in the calculation of the bleeding risk. Previous studies demonstrated that the prevalence of history of bleeding increased the risk of bleeding events. ${ }^{24-26}$ In the previous all-comers study, the prevalence of history of bleeding was approximately $6 \%,{ }^{26}$ whereas in the present study, a prevalence of only $0.6 \%$ was observed. This is a hypothesis-generated study using a database of a randomized controlled trial and bleeding events were site-reported. The PRECISE-DAPT score showed an acceptable predictive performance for short-term bleeding events in spite of a possible event underreporting. In addition, the calculation of the PRECISE-DAPT score is simpler and easier in terms of completing only five variables compared with the other two scores that require many more. Therefore, the PRECISE-DAPT score might be more useful for predicting short-term major bleeding after 30-day DAPT post-PCI in ACS patients compared with the CRUSADE score.

The PRECISE-DAPT score showed a poor calibration in CCS patients although it had a better discrimination than the other scores according to the IDI. One speculation for the explanation of this result is that the PRECISE-DAPT score derivation excluded events in the first 7 days after the index PCI, ${ }^{11}$ whereas they were included in the present study. Therefore, access-site-related bleedings were captured in the bleeding events at 30 days. Historically, the CRUSADE and ACUITY scores also included these accesssite-related bleedings, and while the default access site for PCI has moved from femoral to radial making this site of bleeding less frequent, this should not detract from the fact that a contemporary risk score for short-term bleeding should include procedure-related bleedings.

Finally, the definition of bleeding events in the PRECISEDAPTtrial ${ }^{11}$ was originally thrombosis in myocardial infarction (TIMI) major or minor bleeding from day 7 or later after the index invasive procedure up to 12 months. In the ACUITY trial, ${ }^{6}$ the bleeding definition was TIMI major bleeding within 30 days. In the CRUSADE trial, ${ }^{5}$ the individual bleeding definition (intracranial hemorrhage, documented retroperitoneal bleed, hematocrit drop $\geq 12 \%$ [baseline to nadir], any red blood cell transfusion when baseline hematocrit was $\geq 28 \%$, or any red blood cell transfusion when baseline hematocrit was $<28 \%$ with witnessed bleed) was reported as an in-hospital major bleeding events. This difference in bleeding definition may have affected the relatively low predictive performance of these scores even when assessed in the same patient population. Previous studies demonstrated that all the three scores had a good predictive performance up to 1 year post-PCI in spite of their different bleeding definitions. ${ }^{12}$ However, to date, no validation study of bleeding definitions up to 30 days post-PCI has been reported, and further studies therefore would be needed to verify the differences in the definitions.

\section{Limitations}

The present study has several limitations. First, this study is a post hoc analysis of a neutral randomized controlled study. Inherent subgroup analysis limitations, including the risk of multiple testing, cannot be excluded. Therefore, our findings should be considered as strictly hypothesisgenerating. Second, the bleeding risk and scores were evaluated at the time of randomization, and thus at variance with the PRECISE-DAPT score which excludes the first 7 days. Third, BARC 3 or 5 bleeding was site-reported, as the trial did not have a clinical adjudication committee for serious adverse events due to limited financial resources. However, seven onsite monitoring visits were performed in each participating center, and $20 \%$ of the reported events were checked according to source documents. In addition, the rate of site-reported BARC 3 bleeding in the GLOBAL LEADERS study and the rate of adjudicated BARC 3 bleeding in the GLOBAL LEADERS Adjudication substudy (GLASSY) were similar, a fact that excludes any serious issue of reclassification in bleeding. ${ }^{27,28}$ Fourth, the trial was monitored for event underreporting and event definition consistency. Fifth, the difference in bleeding definitions used might have affected the relatively low predictive performance of bleeding risk scores even in the same patient population. However, to date, no validation study of the bleeding definitions up to 30 days post-PCI has been reported. Finally, the Academic Research Consortium for High Bleeding Risk (ARC-HBR) definition has been developed in 2019, ${ }^{29}$ and validated in a couple of studies. ${ }^{30,31}$ The GLOBAL-LEADERS trial was designed in 2012 and recruited its patients from July 2013 to November $2015 .{ }^{14}$ In the GLOBAL-LEADERS trial, the clinical data of 3 out of 11 major criteria of the ARC-HBR were not collected, and 5 were exclusion criteria.

\section{Conclusion}

The PRECISE-DAPT score showed a similar discriminative capacity for 30-day BARC 3 or 5 bleeding compared with the CRUSADE and ACUITY scores irrespective of clinical presentation, although in CCS patients, it had a poor calibration ability. The PRECISE-DAPT score might be clinically useful in the overall population and ACS patients for the prediction of 
30-day major bleeding post-PCI considering its discriminative and calibration abilities.

\section{What is known about this topic?}

- The PRECISE-DAPT score, which provides a standardized tool for the prediction of mid-term bleeding events during DAPT in an all-comers population, has was developed in 2017.

\section{What does this paper add?}

- The PRECISE-DAPT score showed a similar discriminative capacity for 30-day BARC 3 or 5 bleeding compared with the CRUSADE and ACUITY scores irrespective of clinical presentation, although in CCS patients, it had a poor calibration ability.

- The PRECISE-DAPT score might be clinically useful in the overall population and ACS patients for the prediction of 30-day major bleeding post-PCI considering its discriminative and calibration abilities.

\section{Funding}

The GLOBAL LEADERS study was sponsored by the European Clinical Research Institute, which received funding from AstraZeneca, Biosensors International, and the Medicines Company. The study funders had no role in trial design, data collection, analysis, interpretation of the data, preparation, approval, or making decision to submit the manuscript or publication.

\section{Conflict of Interest}

C.H. reports personal fees from AstraZeneca, during the conduct of the study. P.G.S. reports grants and personal fees from Bayer/Janssen, Merck, Sanofi, Servier, and Amarin; personal fees from Amgen, Bristol Myers Squibb, Boehringer-Ingelheim, Pfizer, Novartis, Regeneron, Lilly, AstraZeneca, grants and personal fees from Servier, outside the submitted work. P.W.S. reports personal fees from Sino Medical Sciences Technology, Philips/Volcano, and Xeltis, outside the submitted work. The other authors have nothing to disclose.

\section{Acknowledgment}

The authors thank the investigators and institutions participating in the GLOBAL LEADERS trial.

\section{References}

1 Vranckx P, Leonardi S, Tebaldi Met al.. Prospective validation of the Bleeding Academic Research Consortium classification in the all-comer PRODIGY trial. Eur Heart J 2014;35(37):2524-2529

2 Hamon M, Lemesle G, Tricot Oet al.. Incidence, source, determinants, and prognostic impact of major bleeding in outpatients with stable coronary artery disease. J Am Coll Cardiol 2014;64 (14):1430-1436
3 Valgimigli M, Bueno H, Byrne RA, et al; ESC Scientific Document Group; ESC Committee for Practice Guidelines (CPG); ESC National Cardiac Societies. 2017 ESC focused update on dual antiplatelet therapy in coronary artery disease developed in collaboration with EACTS: the Task Force for dual antiplatelet therapy in coronary artery disease of the European Society of Cardiology (ESC) and of the European Association for Cardio-Thoracic Surgery (EACTS). Eur Heart J 2018;39(03):213-260

4 Levine GN, Bates ER, Bittl JAet al.. 2016 ACC/AHA guideline focused update on duration of dual antiplatelet therapy in patients with coronary artery disease: a report of the American College of Cardiology/American Heart Association Task Force on Clinical Practice Guidelines. J Am Coll Cardiol 2016;68(10): 1082-1115

5 Subherwal S, Bach RG, Chen AYet al.. Baseline risk of major bleeding in non-ST-segment-elevation myocardial infarction: the CRUSADE (Can Rapid risk stratification of Unstable angina patients Suppress ADverse outcomes with Early implementation of the ACC/AHA Guidelines) bleeding score. Circulation 2009;119 (14):1873-1882

6 Mehran R, Pocock SJ, Nikolsky Eet al.. A risk score to predict bleeding in patients with acute coronary syndromes. J Am Coll Cardiol 2010;55(23):2556-2566

7 Liu R, Lyu SZ, Zhao GQet al.. Comparison of the performance of the CRUSADE, ACUITY-HORIZONS, and ACTION bleeding scores in ACS patients undergoing $\mathrm{PCI}$ : insights from a cohort of 4939 patients in China. J Geriatr Cardiol 2017;14(02):93-99

8 Liu R, Zheng W, Zhao Get al.. Predictive validity of CRUSADE, ACTION and ACUITY-HORIZONS bleeding risk scores in Chinese patients with ST-segment elevation myocardial infarction. Circ J 2018;82(03):791-797

9 Xi S, Zhou S, Wang Xet al.. The performance of CRUSADE and ACUITY bleeding risk scores in ticagrelor-treated ACS patients who underwent PCI. Thromb Haemost 2017;117(11):2186-2193

10 Castini D, Centola M, Ferrante Get al.. Comparison of CRUSADE and ACUITY-HORIZONS bleeding risk scores in patients with acute coronary syndromes. Heart Lung Circ 2019;28(04):567-574

11 Costa F, van Klaveren D, James S, et al; PRECISE-DAPT Study Investigators. Derivation and validation of the predicting bleeding complications in patients undergoing stent implantation and subsequent dual antiplatelet therapy (PRECISE-DAPT) score: a pooled analysis of individual-patient datasets from clinical trials. Lancet 2017;389(10073):1025-1034

12 Choi SY, Kim MH, Cho YRet al.. Performance of PRECISE-DAPT score for predicting bleeding complication during dual antiplatelet therapy. Circ Cardiovasc Interv 2018;11(12):e006837

13 Knuuti J, Wijns W, Saraste Aet al.. ESC Guidelines for the diagnosis and management of chronic coronary syndromes. Eur Heart J 2020;41(03):407-477

14 Vranckx P, Valgimigli M, Jüni P, et al; GLOBAL LEADERS Investigators. Ticagrelor plus aspirin for 1 month, followed by ticagrelor monotherapy for 23 months vs aspirin plus clopidogrel or ticagrelor for 12 months, followed by aspirin monotherapy for 12 months after implantation of a drug-eluting stent: a multicentre, open-label, randomised superiority trial. Lancet 2018;392 (10151):940-949

15 Serruys PW, Takahashi K, Chichareon Pet al.. Impact of long-term ticagrelor monotherapy following 1-month dual antiplatelet therapy in patients who underwent complex percutaneous coronary intervention: insights from the Global Leaders trial. Eur Heart J 2019;40(31):2595-2604

16 Vranckx P, Valgimigli M, Windecker Set al.. Long-term ticagrelor monotherapy versus standard dual antiplatelet therapy followed by aspirin monotherapy in patients undergoing biolimus-eluting stent implantation: rationale and design of the GLOBAL LEADERS trial. EuroIntervention 2016;12(10):1239-1245

17 Marshall A, Altman DG, Holder RL. Comparison of imputation methods for handling missing covariate data when fitting a Cox 
proportional hazards model: a resampling study. BMC Med Res Methodol 2010;10:112

18 DeLong ER, DeLong DM, Clarke-Pearson DL. Comparing the areas under two or more correlated receiver operating characteristic curves: a nonparametric approach. Biometrics 1988;44(03): 837-845

19 Alba AC, Agoritsas T, Walsh Met al.. Discrimination and calibration of clinical prediction models: users' guides to the medical literature. JAMA 2017;318(14):1377-1384

20 Pencina MJ, D’Agostino RB Sr, D’Agostino RB Jr, Vasan RS. Evaluating the added predictive ability of a new marker: from area under the ROC curve to reclassification and beyond. Stat Med 2008;27 (02):157-172

21 Vasan MJPRBDASRBDAJRS. Comments on integrated discrimination and net reclassification improvements - practical advice. Stat Med 2008. Doi: 10.1002/sim.3106

22 Yates JF. External correspondence: decompositions of the mean probability score. Organ Behav Hum Perform 1982;30(01): 132-156

23 Lemeshow S, Hosmer DW Jr. A review of goodness of fit statistics for use in the development of logistic regression models. Am J Epidemiol 1982;115(01):92-106

24 Raposeiras-Roubín S, Faxén J, Íñiguez-Romo Aet al.. Development and external validation of a post-discharge bleeding risk score in patients with acute coronary syndrome: the BleeMACS score. Int J Cardiol 2018;254:10-15

25 van Rein N, Heide-Jørgensen U, Lijfering WM, Dekkers OM, Sørensen HT, Cannegieter SC. Major bleeding rates in atrial fibrillation patients on single, dual, or triple antithrombotic therapy. Circulation 2019;139(06):775-786

26 Simonsson M, Winell H, Olsson Het al.. Development and validation of a novel risk score for in-hospital major bleeding in acute myocardial infarction:-the SWEDEHEART score. J Am Heart Assoc 2019;8(05):e012157

27 Leonardi S, Franzone A, Piccolo Ret al.. Rationale and design of a prospective substudy of clinical endpoint adjudication processes within an investigator-reported randomised controlled trial in patients with coronary artery disease: the GLOBAL LEADERS Adjudication Sub-StudY (GLASSY). BMJ Open 2019;9(03): e026053

28 Franzone A, McFadden E, Leonardi S, et al; GLASSY Investigators. Ticagrelor alone versus dual antiplatelet therapy from 1 month after drug-eluting coronary stenting. J Am Coll Cardiol 2019;74 (18):2223-2234

29 Urban P, Mehran R, Colleran Ret al.. Defining high bleeding risk in patients undergoing percutaneous coronary intervention: a consensus document from the Academic Research Consortium for High Bleeding Risk. Eur Heart J 2019;40(31):2632-2653

30 Natsuaki M, Morimoto T, Shiomi Het al.. Application of the Academic Research Consortium High Bleeding Risk Criteria in an all-comers registry of percutaneous coronary intervention. Circ Cardiovasc Interv 2019;12(11):e008307

31 Ueki Y, Bär S, Losdat Set al.. Validation of bleeding risk criteria (ARC-HBR) in patients undergoing percutaneous coronary intervention and comparison with contemporary bleeding risk scores. EuroIntervention 2020:EIJ-D-20-00052 